\title{
GROWTH OF MULTI-WALLED CARBON NANOTUBES ALONG WITH GRAVITY BY SPRAY PYROLYSIS OF NATURAL PRECURSOR
}

\author{
E. Kanagaraj, P. Mahalingam ${ }^{\bowtie}$, R. Siddharthan and P. Sivakumar \\ Arignar Anna Government Arts College, Namakkal - 637002, Tamil Nadu, India \\ ${ }^{\otimes}$ Corresponding Author: ponmahanano@gmail.com
}

\begin{abstract}
Carbon nanotubes are prepared along the gravity direction in a spray pyrolysis setup over the silica-supported FeCo-Ni catalyst. The silica-supported Fe-Co-Ni catalyst coated over the copper strip is inserted in such a way the coating surface faces downward so that carbon nanotubes can grow along the direction of gravity. Plant-based natural precursor, Pine oil, is used as a carbon source for the preparation of carbon nanotubes by spray pyrolysis method. The effect of precursor flow rate on yield, morphology and structural properties of carbon nanotubes prepared along gravity are discussed. At an optimum flow rate, carbon nanotubes are formed in good yield. The carbon nanotubes synthesized are characterized using appropriate techniques. The scanning electron microscope results show that the synthesized carbon nanotubes are long, straight and well graphitized multi-walled type. The carbon nanotubes diameters are around $50 \mathrm{~nm}$ and the average length $20 \mu \mathrm{m}$. Raman spectroscopy studies revealed that carbon nanotubes are well graphitized multi-walled type with defect-free. A mechanism for the growth of carbon nanotubes along gravity is proposed. Spray pyrolysis of the natural precursor, Pine oil, along with gravity over inversely placed support catalyst material is a promising technique for the growth of long and narrow carbon nanotubes.

Keywords: Carbon Nanotubes, Natural Precursor, Flow Rate, Growth Mechanism, Spray Pyrolysis
\end{abstract}

RASĀYANJ. Chem., Vol. 14, No.4, 2021

\section{INTRODUCTION}

Nanoscience and nanotechnology attract all sections of society due to their prospective application in the field of engineering, health, environment and energy. Carbon nanotubes and other carbon nanostructures are a critical component of this nanotechnology. Carbon nanotubes exhibit unique physical and chemical properties and these properties are generally dependent on their morphology and structure. ${ }^{1}$ The specific properties of these materials is prospective for application in the field of environmental remediation, gas sensor, hydrogen storage, and energy storage devices. ${ }^{2-4}$ The choice of catalyst, precursor, and preparation methods has been extensively studied by several research groups to improve the yield, quality and properties of carbon nanotubes. To date, the synthesis of CNTs with desired properties is reliant on the choice of ideal parameter boundary condition, a specific combination of metal catalysts and appropriate experimental setup. ${ }^{5-6}$ The carbon nanotubes growth properties have been influenced by several factors including precursor and carrier gas flow rate in CVD process. ${ }^{7}$ During growths, carbon nanotubes tend to bow along the flow direction of the precursor and carrier gas. A tip growth mechanism is generally proposed for the growth of carbon nanotubes in CVD process. ${ }^{8}$

In a tip growth mechanism, the catalyst particle drifts from support and move front as carbon nanotubes grow. Owing to heavy metal catalyst particles at the tip of the flexible and lightweight carbon tube, the tube bend during growth and results in entangled carbon nanotubes formation. ${ }^{-11}$ Therefore, as a deviation from the conventional against gravity growth, it is thought to grow carbon nanotubes along the gravity direction. The growth of carbon nanotubes along gravity is reported by Yeh et al group. ${ }^{12-13}$ In this study, they used a mixture of hydrogen and methane as a carbon source and reported that the length of carbon nanotube increases with time. In recent times, as a development towards the green principle, natural renewable precursors are used as an alternative to petroleum products. Following the green

Rasayan J. Chem., 14(4), 2311-2317(2021)

http://doi.org/10.31788/RJC.2021.1446485

This work is licensed under a CC BY 4.0 license. 
chemistry principle, plant-based renewable carbon precursors receive attention to synthesizing carbon nanotubes. ${ }^{14-15}$

Considering these concerns, it is planned to synthesis carbon nanotubes along with the gravity orientation by spray pyrolysis deposition of natural renewable carbon precursor, Pine oil (Botanical name: Pinus Sylvestris oil), with different flow rates.

\section{EXPERIMENTAL}

The precursor flow rate effect on yield and morphology of carbon nanotubes synthesized along the gravity direction from renewable precursors, Pine oil, over silica-supported Fe-Co-Ni catalyst at $650{ }^{\circ} \mathrm{C}$ is studied. A natural precursor, Pine oil, is used as a carbon precursor for the synthesis of carbon nanotubes. The precursor is readily available, cheap and also user-friendly for the spray pyrolysis method. Since these precursors evaporate at a relatively higher temperature, a modified CVD method in the form of spray pyrolysis is adopted for the synthesis of CNTs. Silica supported Fe-Co-Ni is coated over the copper substrate and kept inside the quartz tube in such a way to orient downward to grow carbon nanotubes along the gravity direction. In this setup, the quartz tube kept inside the tubular furnace is flushed with nitrogen to remove air and oxygen inside the tube. Nitrogen gas is continuously passed till end of the experiment to create an inert environment within the quartz tube. The inlet of the quartz tube is attached to the spray gun through which the precursor is showered as mist into the quartz tube. The other end of the quartz tube is attached to the outlet pipe. The quartz tube along with the catalyst kept in the tubular furnace is heated at the rate of $10^{\circ} \mathrm{C} / \mathrm{min}$ till it attains the reaction temperature $650{ }^{\circ} \mathrm{C}$. A suitable precursor flow rate range is selected based on preliminary experiments. 10 to $30 \mathrm{~mL} / \mathrm{hr}$ precursor flow rate range is found to be suitable for carbon nanotubes synthesis. Now, the precursor, $10 \mathrm{~mL}$ for each experiment, is sprayed into the quartz tube at the rate of 10,20 and $30 \mathrm{~mL} / \mathrm{hr}$ for 60 minutes. At the end of the reaction, the furnace is switched off but the flow of nitrogen is continued till the quartz tube attains room temperature. The carbon deposit formed along the gravity direction is collected, weighed and repeatedly washed with 1:1 aqueous nitric acid solution to remove the catalyst particles through the centrifugation process. The carbon deposit obtained is kept in a quartz boat and heated in static air at 400 ${ }^{\circ} \mathrm{C}$ for 1 hour to remove amorphous carbon. The sample is used for characterization. The as-deposited and purified carbon deposits are characterized using appropriate analysis techniques SEM, TGA and Raman.

\section{RESULTS AND DISCUSSION}

Preliminary experiments to fix natural precursor Pine oil flow rate range for growth of carbon nanotubes along gravity are conducted keeping other parameters at optimum condition. The effect of natural precursor Pine oil flow rate 10, 20 and $30 \mathrm{~mL} / \mathrm{hr}$ on the properties of carbon nanotubes grown along gravity direction on silica-supported Fe-Co-Ni catalyst at $650{ }^{\circ} \mathrm{C}$ under nitrogen atmosphere is studied. The carbon deposit is analyzed and discussed on its yield, structure and morphology. The photograph of the carbon deposit grown along the gravity direction is shown in Fig.-1.
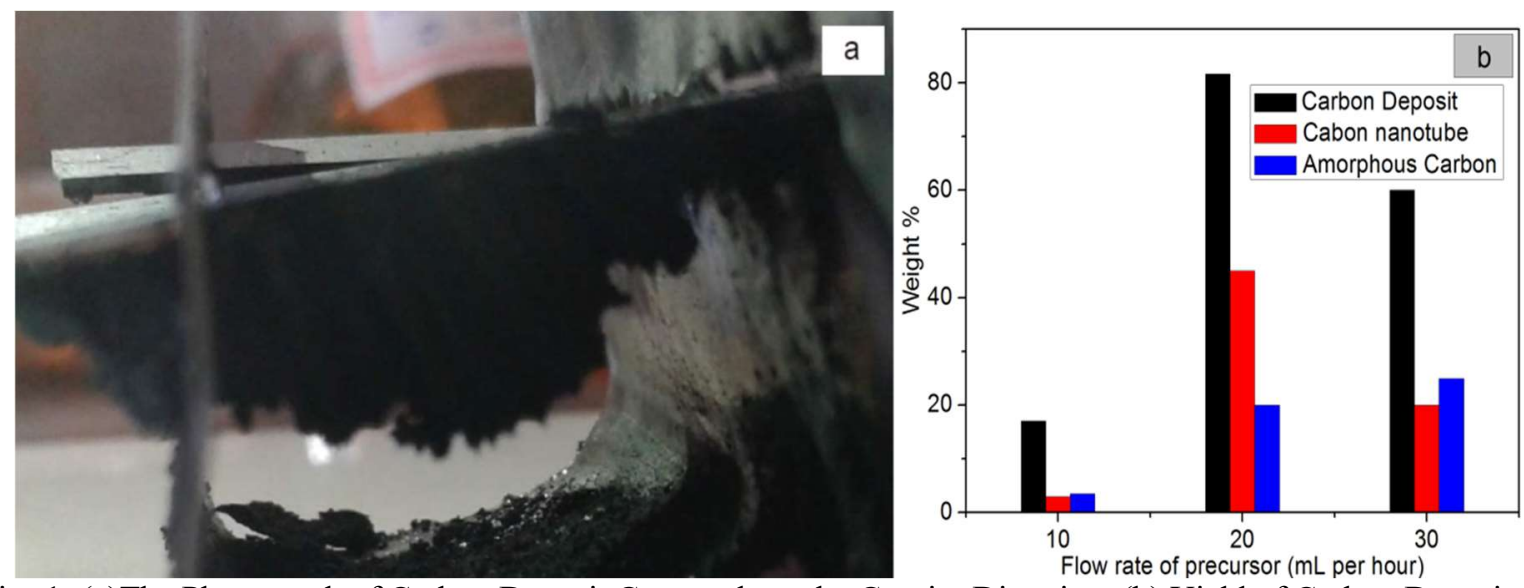

Fig.-1: (a)The Photograph of Carbon Deposit Grown along the Gravity Direction, (b) Yield of Carbon Deposit for a Precursor Flow Rate of 10, 20 and $30 \mathrm{~mL} / \mathrm{hr}$ 
The photographic image (Fig.-1a) illustrates the approach followed to prepare carbon nanotubes along with gravity. Carbon nanotubes growth originated from catalyst layer coated at bottom of the strip and grown downward i.e. along the gravity direction. The clear surface of the non-coated copper strip indicates that carbonization and carbon nanotube formation has not occurred on the surface of the copper strip under the experimental conditions. The carbon deposit observed at the bottom of the setup (Fig.-1a) is due to fly away or detached carbon deposit from the base of the catalyst layer under experimental conditions. The as-grown carbon deposit is analyzed using the thermogravimetric technique (graph not shown). The percentage of amorphous carbon and carbon nanotubes are calculated from the onset decomposition temperature and weight loss occurred. Weight $\%$ of carbon deposit, amorphous carbon and carbon nanotubes are calculated and depicted in figure $1 \mathrm{~b}$ as bar chart. Figure $1 \mathrm{~b}$ shows that increasing precursor flow rate from $10 \mathrm{~mL} / \mathrm{hr}$ to $20 \mathrm{~mL} / \mathrm{hr}$ increases the yield of carbon deposits from $17 \%$ to $81.7 \%$. Further increase of flow rate to $30 \mathrm{~mL} / \mathrm{hr}$ leads to a reduction in the yield (60\%). The observed fact, 10 $\mathrm{mL} / \mathrm{hr}$ precursor flow rate results in low yield, can be attributed to the non-availability of sufficient quantities of precursor vapors for cracking to match with carbon nanotubes growth rate. ${ }^{16}$ The high yield of carbon deposit formed for the precursor feed of $20 \mathrm{~mL} / \mathrm{hr}$ can be attributed to the effective pyrolysis of the precursor at this experimental condition and continuous supply of pyrolyzed carbon at the optimum rate to match with the growth of carbon nanotubes. ${ }^{17}$

Increasing flow rate to $30 \mathrm{~mL} / \mathrm{hr}$ reduces yield to $60 \%$. Several authors have reported similar observations and stated that the yield of carbon nanotubes is affected by the deactivation of catalyst particles at a higher precursor flow rate. ${ }^{18}$ This fact can be attributed to the formation of a carbon layer over the surface of catalyst particles. ${ }^{19}$ The carbon layer over the surface of the catalyst prevents the contact of precursor molecule with the catalyst and thus decreases the pyrolysis of carbon precursor. The low yield of carbon deposit at $30 \mathrm{~mL} / \mathrm{hr}$ flow rate is connected with reduced precursor molecules contact time with the catalyst for pyrolysis and also chances of carbon deposit fly out at higher flow rate.

The SEM image and Raman spectrum were recorded for the carbon nanotubes synthesized along the gravity direction using natural precursor Pine oil feed at $10 \mathrm{~mL}$ per hour over silica-supported Fe-Co-Ni catalysts at $650{ }^{\circ} \mathrm{C}$ is shown in Fig.-2.
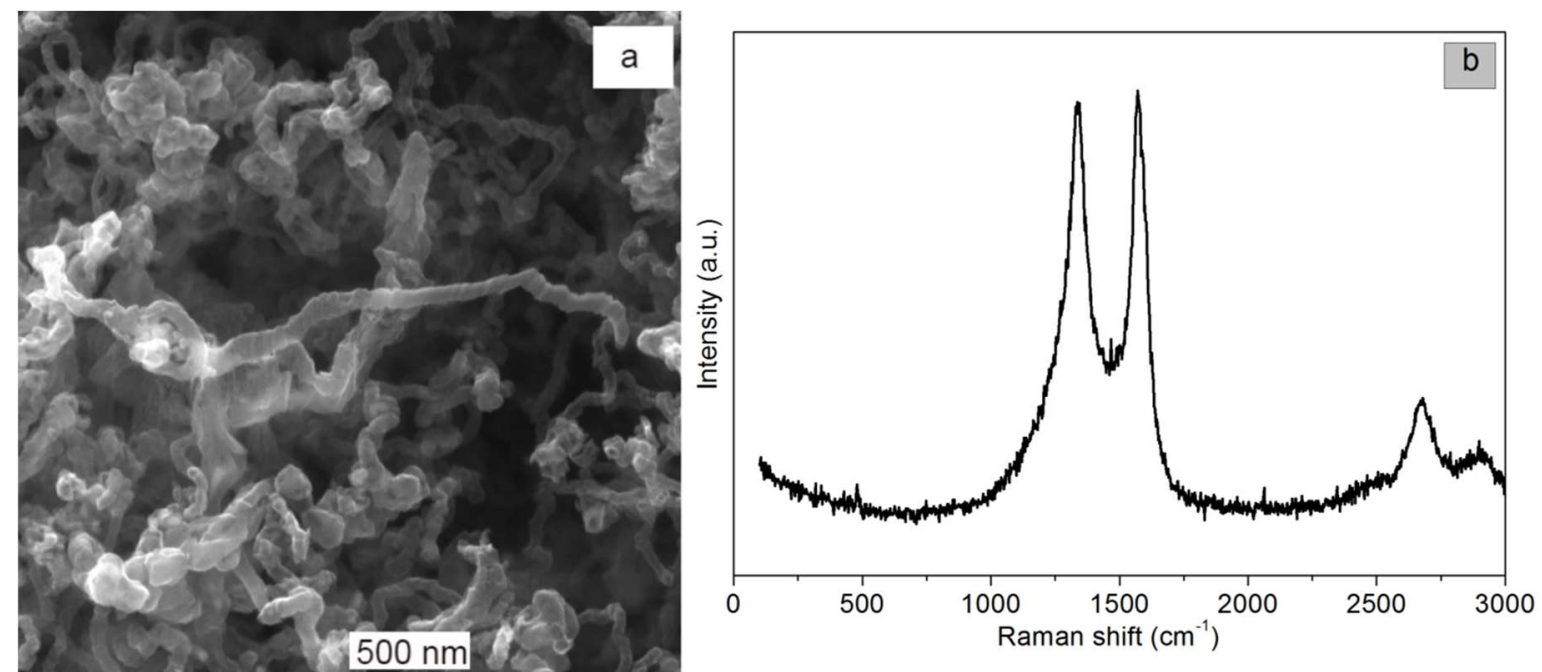

Fig.-2: (a) SEM Image of Carbon Nanotubes synthesized over Silica-supported Fe-Co-Ni Catalyst at $650{ }^{\circ} \mathrm{C}$ for a

Flow rate of $10 \mathrm{~mL} / \mathrm{hr}$ (b) Raman Spectrum of Carbon Nanotubes synthesized over Silica-supported Fe-Co-Ni Catalyst at $650{ }^{\circ} \mathrm{C}$ for a Precursor Flow rate of $10 \mathrm{~mL} / \mathrm{hr}$

The SEM image (Fig.-2a) shows that carbon nanotubes formed are 80-100nm in diameter with the twisted surface. The formation of twisted carbon structure indicates defects in the graphitic layer. The defects on the carbon nanotubes layer and irregular shape of carbon nanotubes formed at this flow rate $(10 \mathrm{~mL} / \mathrm{hr})$ can be attributed to fewer decompositions of the precursor which are unable to cope up with the growth rate of the tube. The crystalline nature of carbon nanotubes is studied using Raman spectrum recorded for the carbon deposit grown along with gravity for precursor flow rate $10 \mathrm{~mL} / \mathrm{hr}$ (Fig.-2b). The G-band 
observed at $1569.9 \mathrm{~cm}^{-1}$ is attributed to the crystalline nature of graphitic layers. The D-band, which represents defects in the layer, is observed at $1340 \mathrm{~cm}^{-1}$. The $\mathrm{I}_{\mathrm{G}} / \mathrm{I}_{\mathrm{D}}$ ratio calculated is 1.03 . This $\mathrm{I}_{\mathrm{G}} / \mathrm{I}_{\mathrm{D}}$ ratio indicates that the carbon nanotubes are formed with moderate graphitization. ${ }^{20}$

The SEM image and Raman spectrum of the carbon deposit grown at $650{ }^{\circ} \mathrm{C}$ for a precursor flow rate of $20 \mathrm{~mL} / \mathrm{hr}$ is shown in Fig.-3.
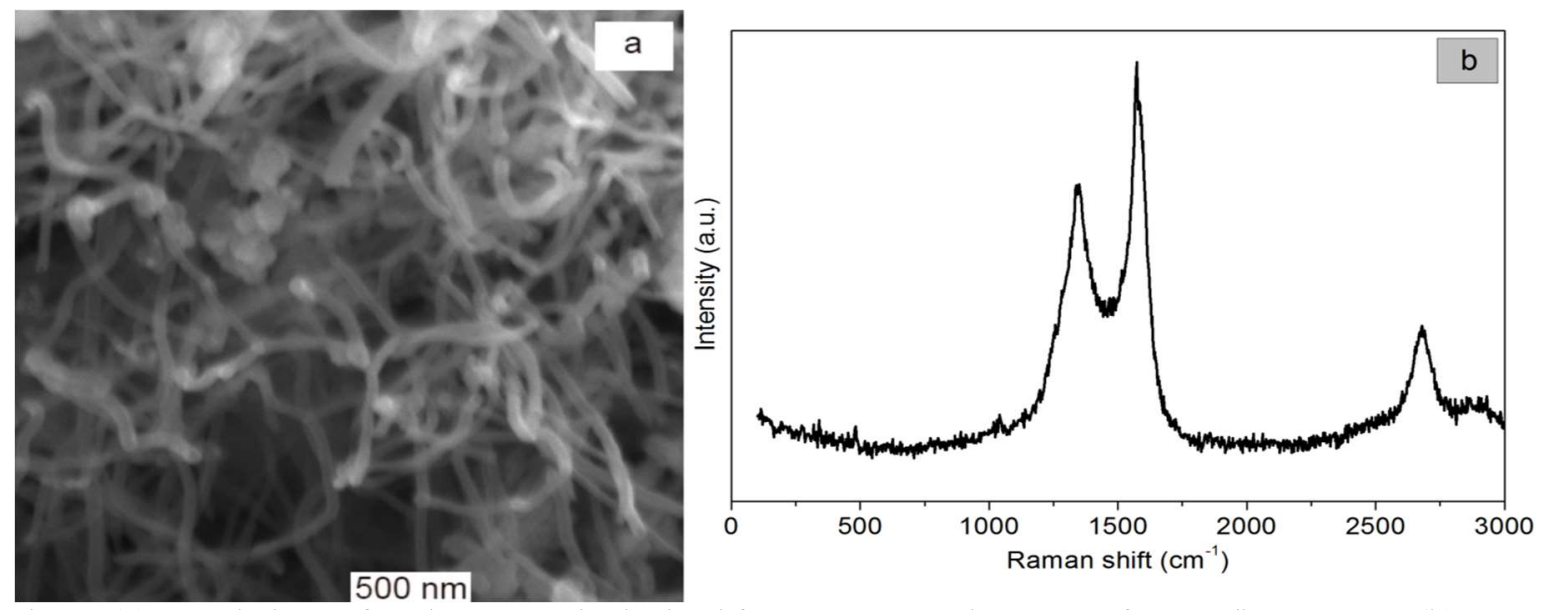

Fig.-3: (a) Morphology of Carbon Deposit obtained for a Precursor Flow Rate of $20 \mathrm{~mL} / \mathrm{hr}$ at $650{ }^{\circ} \mathrm{C}$ (b) Raman Spectrum of Carbon Nanotubes synthesized over Silica-supported Fe-Co-Ni Catalyst at $650{ }^{\circ} \mathrm{C}$ for a Precursor Flow Rate of $20 \mathrm{~mL} / \mathrm{hr}$

The carbon nanotubes are narrow and almost uniform in thickness along the tube length. The average thickness and length of the tubes are $50 \mathrm{~nm}$ and $20 \mu \mathrm{m}$ respectively. The narrow carbon nanotubes formation is attributed to the growth along the gravity direction. The SEM image (Fig.-3a) indicated that smooth-surfaced, narrow and uniform size carbon nanotubes are formed at $20 \mathrm{~mL} / \mathrm{hr}$ precursor flow rate under optimum conditions of other reaction parameters. This shows continuous carbon nanotubes growth occurrence at this precursor flow rate. The crystalline nature of carbon nanotubes prepared for a precursor flow rate of $20 \mathrm{~mL} / \mathrm{hr}$ is studied using the Raman spectrum (Fig.-3b). The characteristic D and G peaks observed at $1335.4 \mathrm{~cm}^{-1}$ and $1572.6 \mathrm{~cm}^{-1}$ are attributed to defects and crystalline graphitic layers respectively. The $\mathrm{I}_{\mathrm{G}} / \mathrm{I}_{\mathrm{D}}$ ratio of 1.32 indicates that the carbon nanotubes graphitic layers are well crystallized and almost free from defects and amorphous carbon.

The effect of precursor flow rate $(30 \mathrm{~mL} / \mathrm{hr})$ on the morphology of carbon nanotubes grown along gravity direction at $650{ }^{\circ} \mathrm{C}$ over silica-supported Fe-Co-Ni catalysts is studied. A typical SEM image and Raman spectrum of the carbon deposit are shown in Fig.-4.
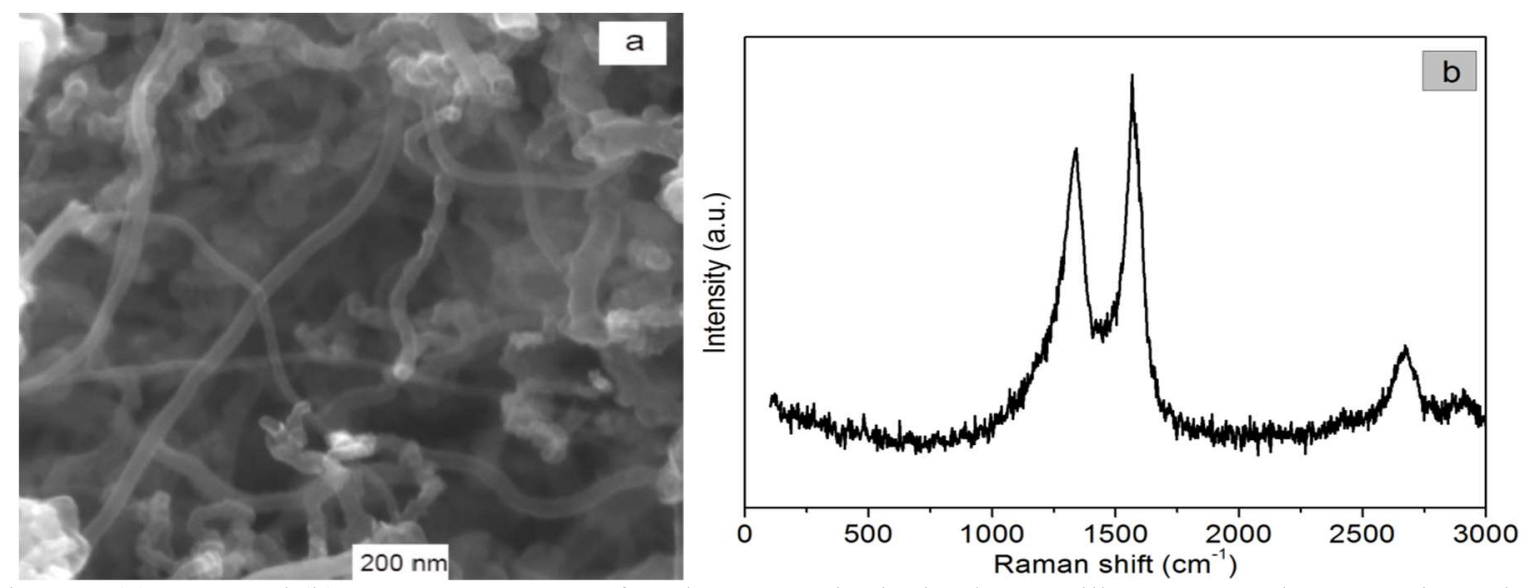

Fig.-4: (a) SEM and (b) Raman Spectrum of Carbon Deposit obtained over Silica-supported Fe-Co-Ni Catalyst at $650{ }^{\circ} \mathrm{C}$ for the Precursor Flow Rate of $30 \mathrm{~mL} / \mathrm{hr}$. 
The morphology of carbon nanotubes is characterized using the SEM image (Fig.-4a). Increasing precursor flow rate to $30 \mathrm{~mL} / \mathrm{hr}$ has resulted in the formation of amorphous carbon together with carbon nanotubes. The thickness of carbon nanotubes is found to be $70 \mathrm{~nm}$. Moreover, the surface of the tubes is not smooth and narrow. It is attributed to the fact that the catalyst particles are getting deactivated as the precursor vapor increased under a high precursor flow rate. The catalysts get deactivated due to the formation of carbon layers which occur under the condition of a larger quantity of carbon produced by pyrolysis than the assimilation of carbon by catalysts for the growth of carbon nanotubes. These unassimilated carbon forms layer over the surface of the catalyst. Esconjauregui et al stated that whichever metal acts as a catalyst is deactivated under an increased partial pressure of any of the carbon sources, probably due to catalyst encapsulation by amorphous carbon or graphitic layers. ${ }^{21}$ The results obtained for a different flow rate of precursor Pine oil agree with literature reports. ${ }^{22}$

The carbon deposit obtained over silica-supported Fe-Co-Ni catalysts at $650{ }^{\circ} \mathrm{C}$ for a precursor flow rate of $30 \mathrm{~mL} / \mathrm{hr}$ is characterized using Raman spectroscopy to evaluate the graphitization of carbon in deposit. Raman spectrum obtained for the carbon deposit is depicted in Fig.-4(b). The Raman spectral analysis provides information about the crystalline nature of carbon through the characteristic D and G peaks. The appearance of $\mathrm{D}$ and $\mathrm{G}$ peaks at $1335 \mathrm{~cm}^{-1}$ and $1567 \mathrm{~cm}^{-1}$ respectively with $\mathrm{I}_{\mathrm{G}} / \mathrm{I}_{\mathrm{D}}$ value of 1.26 indicates that the graphitic layers of carbon are defective and also contain more amorphous carbon. The absence of RBM peak for the carbon deposit prepared indicates that the single-walled carbon nanotubes are not formed under the experimental conditions.

Due to the complex nature of carbon nanotube growth, to date, no acceptable single mechanism is established. Various growth mechanisms are proposed based on the condition in which carbon nanotubes are prepared. One of the mechanisms accepted in general for the growth of carbon nanotubes in CVD process is a vapor-Liquid-Solid (VLS) mechanism. ${ }^{23}$ Since, carbon nanotubes are synthesized along with gravity in this work, a growth mechanism is proposed based on the results obtained (Fig.-5).

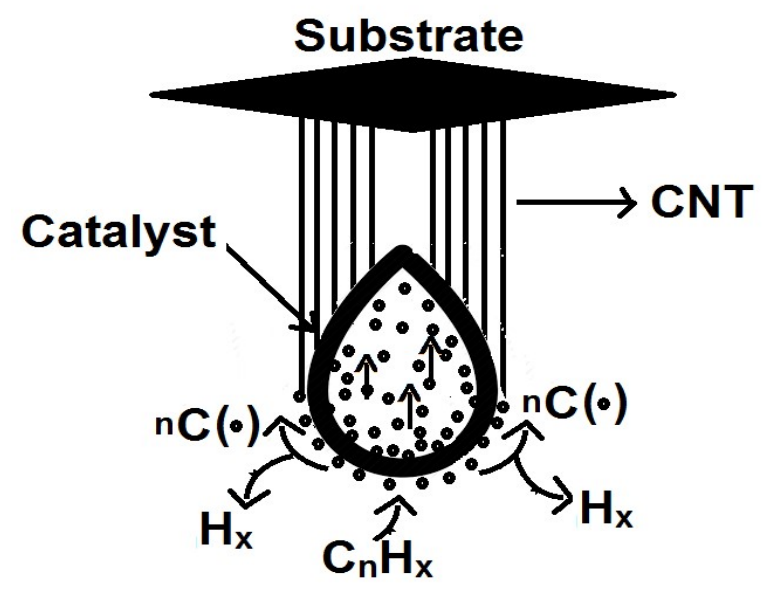

Fig.-5: Mechanism for Growth of Carbon Nanotube along withGravity

The catalysts which are supported on oxide materials are the main cause for pyrolysis of the precursor at a lower temperature. The interaction between the catalyst and support plays a major role in deciding whether carbon nanotubes grow by tip growth or bottom growth. The bottom growth of carbon nanotubes follows when the interaction between metal and support is strong and tip growth if the interaction is weak. Tip growth is the one of accepted mechanisms for carbon nanotube growth in CVD process. ${ }^{24}$ The catalyst in the form of liquid drop at the tip of carbon nanotubes moves straight along the gravity. When the rate of pyrolysis of the precursor and assimilation of carbon produced by catalysts are appropriate during the growth of carbon nanotubes, a narrow and uniform thick tube is formed. The SEM image (Fig.2a) indicates the existence of catalyst particles at the tip of carbon nanotubes. Therefore, it is proposed that the carbon nanotubes grow through tip growth.

The precursor vapors, when coming in contact with the catalyst at the appropriate temperature, get pyrolyzed. The carbon produced due to pyrolysis dissolves in the metal by forming metal carbides. ${ }^{25}$ Once the carbon content in the liquid metal drop exceeds its solubility, it drifts out of the metal liquid drop and 
is combined to form a tube-like network. The tube formation is ascribed to the spherical shape of the liquid metal drop. The crystallization of carbon as a tube depends on the size, shape of a liquid metal drop, the quantity of carbon dissolved in the catalyst metal as well as the rate at which carbon is ejected from the metal liquid drop. Higher the rate at which carbons are drifted out from liquid metal drop than the rate of crystallization, amorphous carbon are formed while, if it is low, dangling bonds are formed on the surface of the layer. The diffusion of carbon in a metal can occur either on the surface or through the bulk of the liquid metal. If carbon diffuses through the outer surface only, then a large inner diameter with few walls of carbon nanotubes is expected. While, if the diffusion of carbon occurs through the bulk of the liquid metal, multi-walled carbon nanotubes with smaller inner diameters would be produced. ${ }^{26}$ In this work, under the experimental condition, bulk diffusion of carbon through the catalyst is proposed as multi-walled carbon nanotubes with smaller inner diameters are formed.

Even though growth along gravity leads to lengthy and narrow carbon tube formation, the precursor and carrier gas flow rate affect its morphology and structure. It is found that the effect of gravity dominates the disturbance caused by the precursor when passed at the rate of $20 \mathrm{~mL} / \mathrm{hr}$. The high flow rate of precursor $(30 \mathrm{~mL} / \mathrm{hr})$ dominates the gravity force thus, carbon nanotubes are entangled and also carbon deposits fly out from the surface of the substrate. At a lower flow rate $(10 \mathrm{~mL} / \mathrm{hr})$ the gravity dominates the force exerted by the flow of precursor vapor, due to low carbon precursor vapor density, amorphous and defective surface carbon nanotubes are formed. This work suggests that the synthesis of multi-walled carbon nanotubes with desired quality requires maintaining the precursor flow rate at an appropriate level under optimum conditions of other reaction parameters.

\section{CONCLUSION}

Confirming the green chemistry principles, natural renewable precursor Pine oil (Botanical name: Pinus Sylvestris oil) is explored for the growth of multi-walled carbon nanotubes along with gravity in a spray pyrolysis setup. Natural renewable precursor, Pine oil, can be used to synthesis multi-walled carbon nanotubes along gravity direction over silica-supported Fe-Co-Ni catalysts using spray pyrolysis method. The effect of the flow rate of the precursor on the yield, morphology and properties revealed that the optimum precursor flow rate under the experimental condition produced narrow, long and well graphitized multi-walled carbon nanotubes in good yield. A mechanism for the growth of carbon nanotubes along gravity is suggested.

\section{ACKNOWLEDGEMENT}

The authors acknowledge UGC for the instrumental facility (MRP-5394/14 (SERO/UGC) March 2014). The author also thanks Gandhigram University, Dindigul, Alagappa University, Karaikudi, India for material characterization analysis.

\section{REFERENCES}

1. N.J. Coville, S. D. Mhlanga, E.N. Nxumalo and A. Shaikjee, South African Journal of Science, 107(3-4), Art. \#418, 15 pages (2011), http://dx.doi.org/10.4102/sajs.v107i3/4.418

2. N. Gupta, S. M. Gupta and S. K. Sharma, Carbon Letters, 29(5), 3(2019), https://doi.org/10.1007/s42823-019-00068-2

3. S. Kalaiselvan, K. Anitha, P. Shanthi, P.S. Syed Shabudeen and S. Karthikeyan, Rasayan Journal of Chemistry, 7(4), 333(2014)

4. B. Murugesan, A. Sivakumar, A. Loganathan, P. Sivakumar, Journal of the Taiwan Institute of Chemical Engineers,71, 364( 2017), https://doi.org/10.1016/j.jtice.2016.11.020

5. S. Karthikeyan, P. Mahalingamand M. Karthik, Journal of Chemistry, 6(1), 1(2009), https://doi.org/10.1155/2009/756410

6. E. Kanagaraj, P. Mahalingam, R. Siddharthan and C. Sathishkumar, Asian Journal of Chemistry, 33(5), 989(2021), https://doi.org/10.14233/ajchem.2021.23118

7. Z. Zhu, Y. Bai, N. Wei, J. Gao, S. Sun, C. Zhang and F. Wei, Nano Research,13,1988(2020), https://doi.org/10.1007/s12274-020-2898-2

8. M. Kumar and Y. Ando, Journal of Nanoscience and Nanotechnology, 10(6), 3739(2010), https://doi.org/10.1166/jnn.2010.2939 
RASĀYAN J. Chem.

Vol. 14 | No. 4 |2311-2317| October- December | 2021

9. M. Endo, K. Takeuchi, S. Igarashi, K. Kobori, M. Shiraishi and H.W. Kroto, Journal of Physics and Chemistry of Solids, 54(12), 1841(1993), https://doi.org/10.1016/0022-3697(93)90297-5

10. R. Iuliana, H. Yael and C. David, Nanotechnology, 15, 473(2004), https://Doi.org/10.1088/0957$\underline{4484 / 15 / 5 / 011}$

11. K.A. Shah and B.A. Tali, Materials Science in Semiconductor Processing, 41, 67(2016), https://doi.org/10.1016/j.mssp.2015.08.013

12. C.M. Yeh, M.Y. Chen, J.S. Syu, J.Y. Gan and J. Hwanga, Applied Physics Letters, 89, 033117 (2006), https://doi.org/10.1063/1.2228068

13. C.M. Yeh, M.Y. Chen, J.Y. Gan, J.H. Wang, C.D. Lin, T.Y. Chao and Y.T. Cheng, Nanotechnology, 18, 145613 (2007), https://doi.org/10.1088/0957-4484/18/14/145613

14. S. Karthikeyan and P. Mahalingam, International Journal of Green Nanotechnology: Physics and Chemistry, 2, 39(2010), https://doi.org/10.1080/19430876.2010.532421

15. S. Vivekanandhan, M. Schreiber, S. Muthuramkumar, M. Misra and A. Kumar Mohanty, Journal of Applied Polymer Science, 134, 44255(2017), https://doi.org/10.1002/app.44255

16. N. Gilbert, S. Matteo, P. Desiree, O. Kevin, H.A. John, M. Eric, R. Christopher, G. Philip and T. Carl, Carbon, 49(2011), https://doi.org/10.1016/j.carbon.2010.10.018

17. G. Chen, R.C. Davis, H. Kimura, S. Sakurai, M. Yumura, D.N. Futaba and K.Hata, Nanoscale, 7(19), 8873(2015), https://doi.org/10.1039/c5nr01125f

18. V. Jourdain and C. Bichara., Carbon, 58, 2(2013), https://doi.org/10.1016/j.carbon.2013.02.046

19. Y. Homma, Y. Kobayashi, T. Ogino, D. Takagi, R. Ito, Y.J. Jung and P.M. Ajayan, The Journal of Physical Chemistry B, 107(44), 12161(2003), https://doi.org/10.1021/jp0353845

20. R.A. DiLeo, B.J. Landi and R.P. Raffaelle, Journal of Applied Physics, 101, 064307(2007), https://doi.org/10.1063/1.2712152

21. E. Santiago, W. Caroline and M. Karen, Carbon, 47, 6592009, https://doi.org/10.1016/j.carbon.2008.10.047

22. V.S. Angulakshmi, S. Karthikeyan and P.S. Syed Shabudeen, Rasayan Journal of Chemistry, 8(1), 1(2015)

23. E.F. Kukovitsky, S.G. L'vov and N.A. Sainov, Chemical Physics Letters, 317(1-2), 65(2000), https://doi.org/10.1016/S0009-2614(99)01299-3

24. J.C. Charlier, H. Amara and Ph. Lambin, ACS Nano, 1(3), 202(2007), https://doi.org/10.1021/nn700049q

25. C. Emmenegger, J.M. Bonard, M. Philippe, P. Sudan, A. Lepora, B. Grobety, Z. Andreas and S. Louis, Carbon, 41, 539(2003), https://doi.org/10.1016/S0008-6223(02)00362-7

26. S.C. Tseng, T.C. Lee and H.Y. Tsai, Diamond and Related Materials, 96, 112(2019), https://doi.org/10.1016/j.diamond.2019.04.020

[RJC-6485/2021] 